\title{
CYBER-MIXMECHATRONICS MIX CONCEPTS, INTEGRATED IN COBOTIC PLATFORMS FOR THE SMART INDUSTRY (4.0) AND THE DIGITIZED ENTERPRISE
}

\author{
Gheorghe Ion Gheorghe $e^{1,2}$ \\ ${ }^{1}$ National Institute of Research and Development in Mechatronics and Measurement Technique, Bucharest and \\ ${ }^{2}$ Correspondent member of Technical Sciences Academy of Romania \\ Sos. Pantelimon 6-8, Romania \\ geocefin@yahoo.com
}

\begin{abstract}
The scientific paper focuses on a synthesis, the architecture: industry 4.0 and the digital enterprise, intelligent technological change, intelligent societal change and business paradigm shift, new intelligent industry opportunities (4.0) created by the development of mechatronics and cyber-mixmechatronics, information ensures the satisfaction of man's spiritual needs, computer science adds value to everything, computer science culture, the vision of INCDMTM - Romania in the intelligent domain research and innovation "Inthernet of Things - IoT", integration in COBOT technology platforms and the INCDMTM - Romania mission for mechatronic and cybermixmechronic concepts and constructions integrated in cobot technology platforms - for Intelligent Industry (4.0) and the Digitized Enterprise.

The scientific paper concludes the impact of enterprise and intelligent industry digitization through mechatronic and cyber-mixmechronic integrated systems that are or are to be implemented in different industrial sectors - automotive, aerospace, agro-industry, medicine, etc. in Romania and their digitization strategy at national and European level.
\end{abstract}

Keywords: Concepts Cyber-mixmechatronics; COBOT Tehnology Platforms; Digital Enterprise; Intelligent Industry (4.0).

\section{Industry 4.0 and Digital Enterprise}

The Industry 4.0 includes the digitization of production processes based on systems that communicate with each other in an autonomous way along the value chain. This Industry 4.0 considers the potential of the initiative and changes in the business paradigm and the impact of this transformation.

The Industry 4.0 includes, among others:

- technological change;

- societal changes and

- changes in the business paradigm,

as an industrial policy at EU level to support Member States and businesses in the transformation needed to connect and integrate digital technologies with industrial products and services.

Thus, the three key dimensions of consistent change for Industry 4.0 are explored through: technological, social and business paradigm.

Essentially, Industry $\mathbf{4 . 0}$ describes the organization of intelligent production processes based on technologies and products / systems that communicate autonomously with each other along the value chain: a smart factory model of the future where computer-driven systems monitor physical processes, create a virtual copy of the physical world and make decentralized decisions based on self-organizing systems.

The Industry 4.0 concept takes into account the digital growth of manufacturing industries where physical objects are perfectly integrated into the information network, allowing for decentralized production and real-time adaptation in the future.

Industry 4.0 has been developed by developed European countries to create a coherent policy framework for maintaining industrial competitiveness and productivity and for integrating Internet objects, Internet services, Industrial Internet, advanced manufacturing, and intelligent manufacturing.

For Industry 4.0 to be succeed, some key requirements such as system standardization, platforms, protocols, changes in work organization, reflecting new business models, digital security and know-how protection, availability of suitably qualified workers, research and investment, and a legal framework (EU common) to support the 
dissemination of Industry 4.0 on the internal and external market.

As the industry's 4.0 Implementation horizon is to have "pilots" in the pipeline and their full implementation, starting in 2025.

In support of Industry 4.0, the policy approach is to develop new core markets into a dual strategy, where:

- technology and services in Industry 4.0 can be sold, and

- production and other products can be sold more easily due to increased productivity and competitiveness.

For Industry 4.0, the dimensions of change are relevant: technological change, social change, and changes in the business paradigm.

Technological change approach, digitization is a determinant of changes throughout the value chain and while many enterprises recognize the need for adaptation, much less, especially among SMEs, are prepared for it. There are significant challenges (costs and risks) for businesses in terms of digital security in: the protection of intellectual property, personal data and privacy; system design and operability; environmental protection and health and safety.

Social change approach, there is little awareness of Industry 4.0. Although there is a gap in skills (as well as a discrepancy in the desire to adapt to the digital single market), qualification requirements to adapt to Industry 4.0 are much higher. The provision of skills and capabilities in Industry 4.0 across the EU is uneven, which may lead to increased concentration and competition between existing centers.

Business paradigm change approach, there are difficulties for SMEs to participate in the Supply Chain industry 4.0 (costs, risks, reduced flexibility and strategic reduction, independence). The public sector can play a role in creating an ecosystem that will help SMEs move to Industry 4.0.

Standardization remains a major challenge in the widespread implementation of Industry 4.0.
The question here is whether Industry 4.0 will consolidate national industries or EU industry, or whether it is more necessary to maintain its position or whether it will inevitably shift to new emerging economies such as China through the international dissemination of technology by multinationals remains a response to it.

Public sector intervention could take different forms but the most promising seems to be supporting research at EU and Member State level and coordinating initiatives across the EU, through a platform and to illustrate the good practices of initiatives in some Member States that others could follow. In order to maximize value added, initiatives should only go beyond technical issues and the production sector and should reflect the differences in the economic structures of the Member States.

In conclusion are recommended:

(a) the review of existing measures for Industry 4.0 and taking into account the most important issues (skills, migration, change of business business models, clusters, cross-border business collaboration programs, IT security and standards, etc.).

(b) adopting new measures to identify gaps at EU and Member State level to monitor the latest developments, fund research and support SMEs, raise public awareness of challenges and opportunities, support the development of a framework including standards and role coordination.

\section{The Oportunities of Intelligent Industry (4.0) Created by the Development of Cyber- MixMechatronics}

In brief are presented the opportunities in the Intelligent Industry (4.0) created by the development of Cyber-MixMechatronics, architecture for $2009 \div 2018$

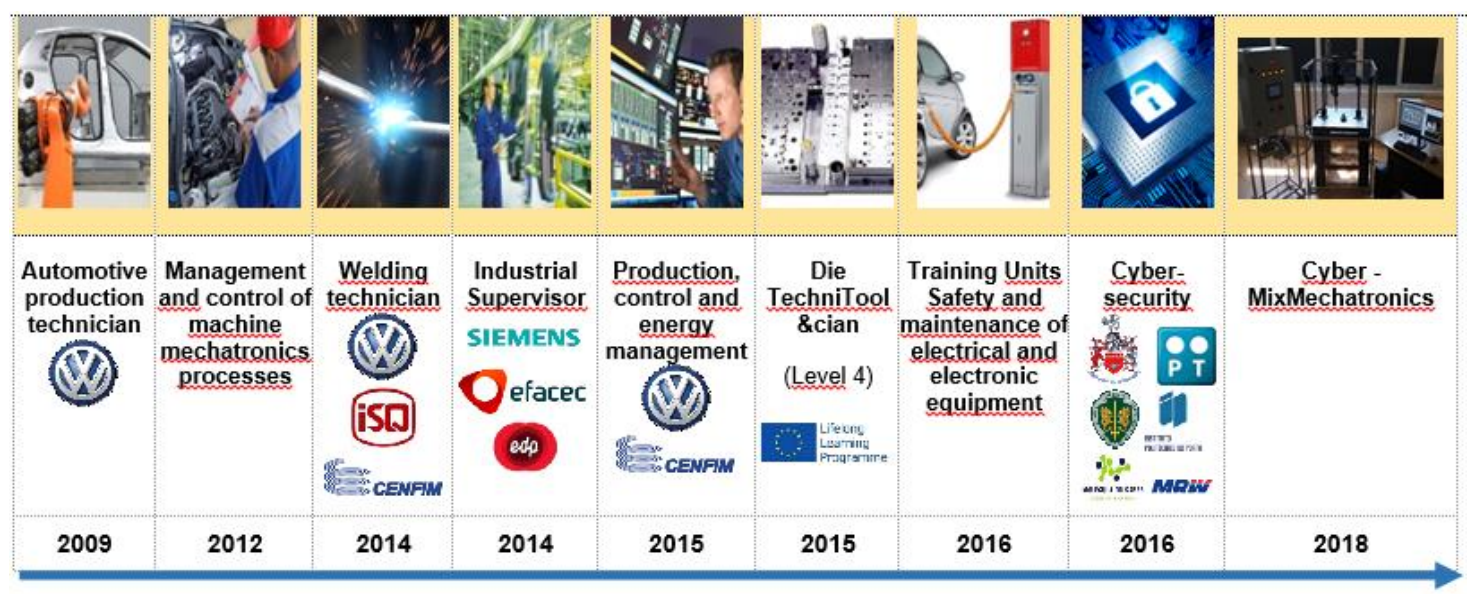


Cyber-mixmechatronics technology puts the focus on information, which is the tonal component in relation to material and energy.

This position of the information is motivated (by the Japanese) by the following arguments:

- information ensures the satisfaction of the spiritual needs of human;

- only computer science increases the added value of all things;

- computer science means culture.

Promoting information links in the technical systems structure ensures flexibility and reconfigurability.

Quantitative and qualitative assessment of information is an essential issue in education, research and production.

Information is equally important in medicine, literature, art, music, sports, etc.

\section{The Author's Vision in Innovation of domain "Internet of Things - IoT" and its Integration into Intelligent Cobotic Platforms}

Research and Innovation in Romania are due to the advanced development of information and communication technology, along with integrative mechatronics that generate more and more objects / objects integrated with sensors and communication capability with other objects / things that transform the physical world into a matrix informativeknowledge system.

The Internet of Objects approach - IoT allows today that things / objects in our environment are active participants who share information with other actors or members of the network, wired / wireless, using the same Internet Protocol-IP protocol that connects the Internet. Thus, objects / things are able to recognize events and changes around them and act and react in a quasi-autonomous way without human intervention.

In this exposed context, the approaches in research, development and innovation create an "intelligent planet," where physical, digital and virtual worlds converge to create smart environments that can make energy, transport, cities and many other intelligent domains.

The development of some generic technologies such as "nano electronics", "communications", "sensors", "smart phones", "embedded systems", "cloud computing and software" will be among the key to supporting future innovations of IoT and cyber mixmechatronics products, which influences many industrial sectors.

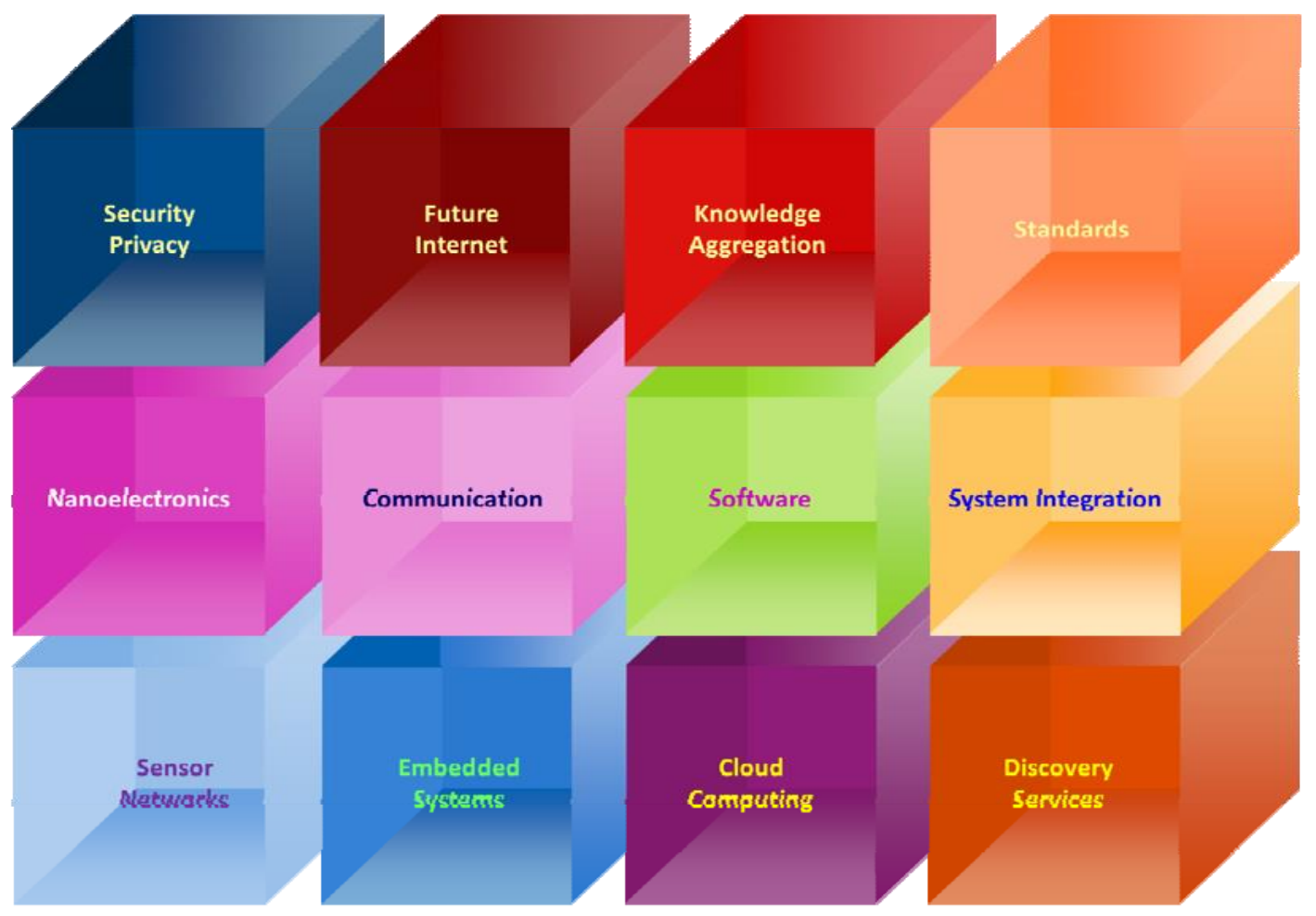

Internet of Things - Enabling Technologies 
In Romania, many projects and initiatives address technologies and knowledge about the Internet of Things and Cyber-Mix Mechatronics.

Thus, the integration of knowledge in this context is conceptualized as the process through which disparate, specialized knowledge, located in several projects in Romania (and at the level of the European Union), are increasingly combined, applied and assimilated. The author and INCDMTM-Bucharest is developing inovation in IoT on the Internet of Things that aims at defining IoT technology and developing research challenges at national (and European) level with a view to the global development of multidisciplinary science. Motivation for the Internet of Things is to address the high potential of IoT-based capabilities in Romania (as well as in Europe) - to coordinate / encourage the convergence of ongoing activities on the most important issues - to build a broad consensus based on modalities IoT in Romania (as in Europe).
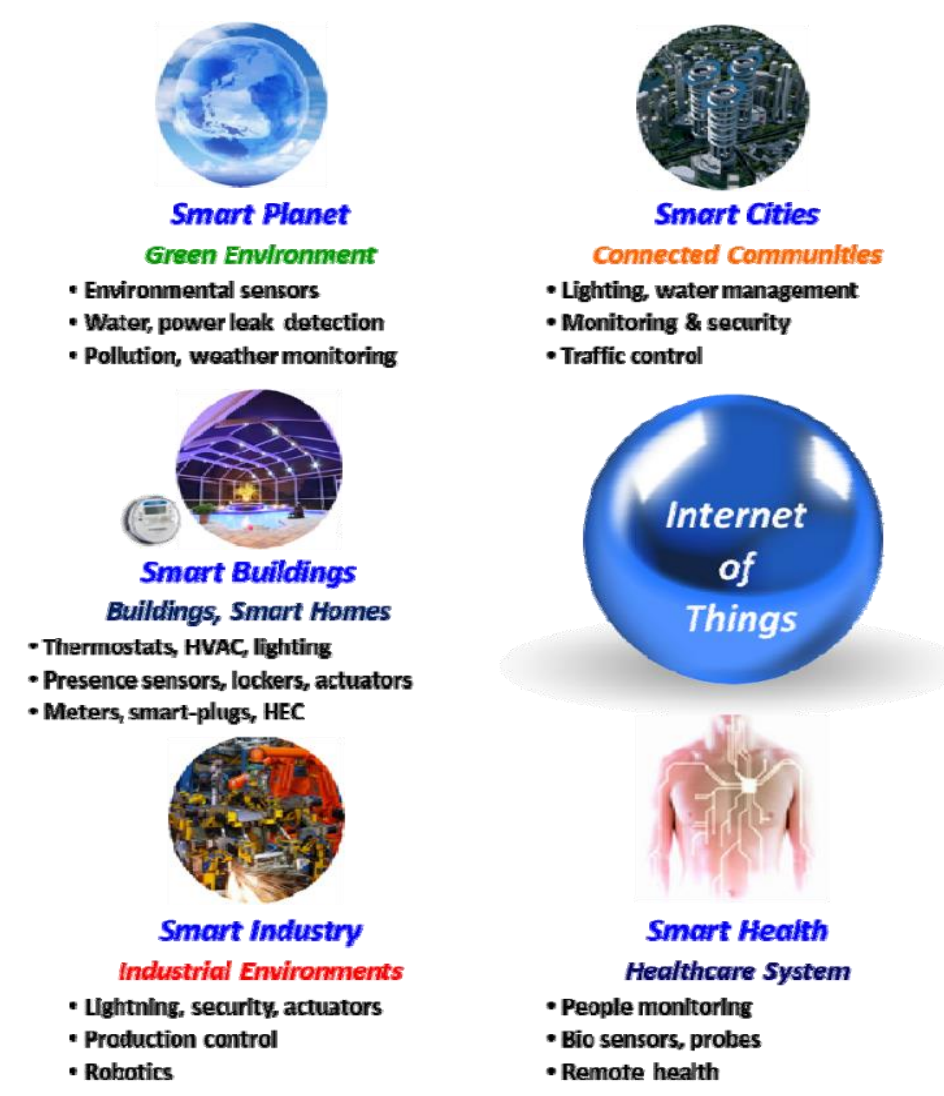

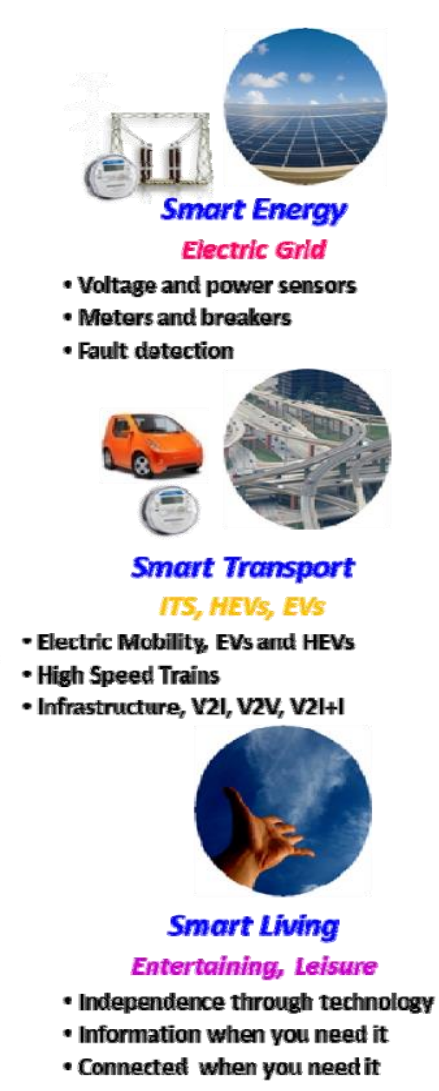

The applications of the Internet energy are connected through The Future Internet and "Internet of Things, allowing for smooth and secure interactions of intelligent systems embedded over heterogeneous communication infrastructures.

Thus, developments in the global environment and developments in Romania's policy, science and technology policy and intelligent industrial policy and digitized enterprise converge to the objective of supporting these links at national level (in Romania).

That is why the author and INCDMTM to achieve real coordination and cooperation in Romania, as well as at European level, is to support the national link of the scientific groups with other national scientific and innovation networks and research based on the intelligent industrial application research, digitized enterprise.

The creation of IoT national networks will allow better coordination of IoT knowledge-producing projects at national level with cross-country interdependencies and cooperation at European level through integration and interconnection.

This concept of linkage will contribute to the consolidation and replication of the success factors achieved through specific IOT projects and will be an instrument that will help to promote the exchange of ideas, solutions, results and their validation among research projects at national level and European.

Integration of IoT with mechatronic and cybermixmechronic systems for telemetry, telecontrol, telemonitoring and teleconfiguration processes have led to new concepts of intelligent system systems.

The following are the concepts of COBOT technology platforms for different applications in intelligent industrial environments.

In the architecture below, the "basic principle for intelligent agriculture" is presented: 


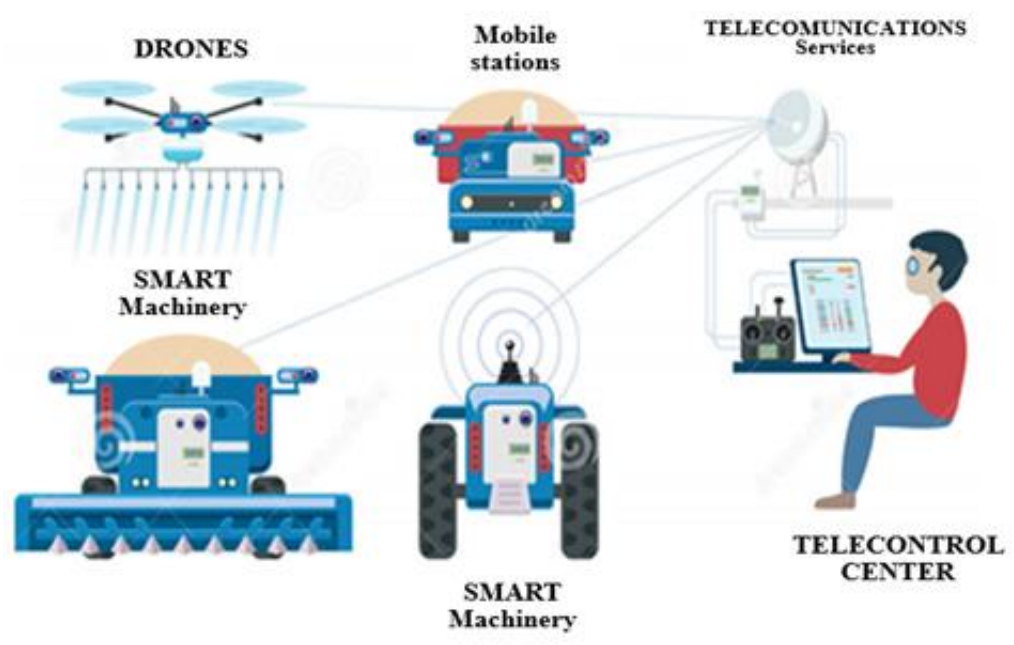

Robotics and more and more integrated technical innovation have boosted the agricultural world for many years. Of these, drones, robots that perform more tasks, such as weeding, milking, sowing, etc. These allow, in addition to suppressing the notion of "hardness" related to certain tasks, to collect accurate and useful data. They promote farm productivity and optimization. These autonomous and digital tools can, for example, be used to reduce the use of agricultural inputs and hence to restore their environmental impact.

Therefore, technological improvements appear for agriculture as a tool and as a key development. And this, as well as environmental aspects such as traceability of products and improvement of operator activity, are defining.

The architecture presented below, represents "Industry 4.0 and Digital Enterprise as a interconnected reality of industrial production flows":

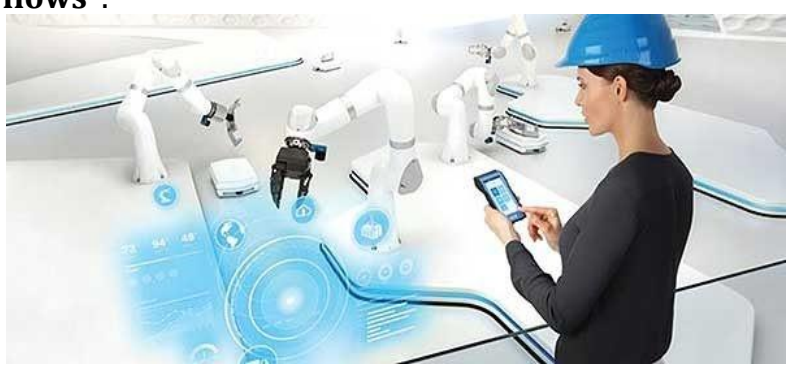

Interconnected production and control processes in industrial environments that operate complex machines will determine the future of Industry 4.0 that contributes to the implementation of dynamic, real-time and self-sustaining processes by providing active and passive detection and interconnection solutions.

The interconnection of individual production steps means that these steps can be combined in any way they need, with maximum traceability of information and field status.
This enables us to create fully observable production lines in real time, efficiency and flexibility being the main features of intelligent factories.

\section{The Vision and the Mission of the Author and INCDMTM Bucharest for Cyber-Mixmechatronics Concepts Integrated in Cobotic Platforms for Intelligent Industry (4.0) and Digital Enterprise}

Thus, there are presented some concepts of cobotic platforms, for different fields and industries, designed in original solutions of the author of this scientific paper:

- in figure 1: The COBOT Technology Platform for Integrated Verification and Control Processes in the Digitized Enterprise and Industry 4.0 in the MixMecatronic field.

The COBOT technology platform for integrated verification and control processes is logically structured on the intelligent mecatronic and cybermixmecatronic architecture (4D / digital ultraprecision measuring system / control and control system / pneutronic antivibration system / etc) + architecture Mechatronic system for leakage of casting parts (casting system / casting / casting system / instrument pneumatic air / plunger system which ensures the closure of all the holes of the casting and thus ensures the space enclosed in the pneumatic / Input / Output Actuator / Mechatronic Mechatronic Metering System / Leakage Mechatronic System Checkpiece (after result display) / Mechatronic Operating and Leak Test System optical signaling system leakage checking process - system operation - system malfunction alarm - total system / pneumatic system / system power blocking system the architecture of the 


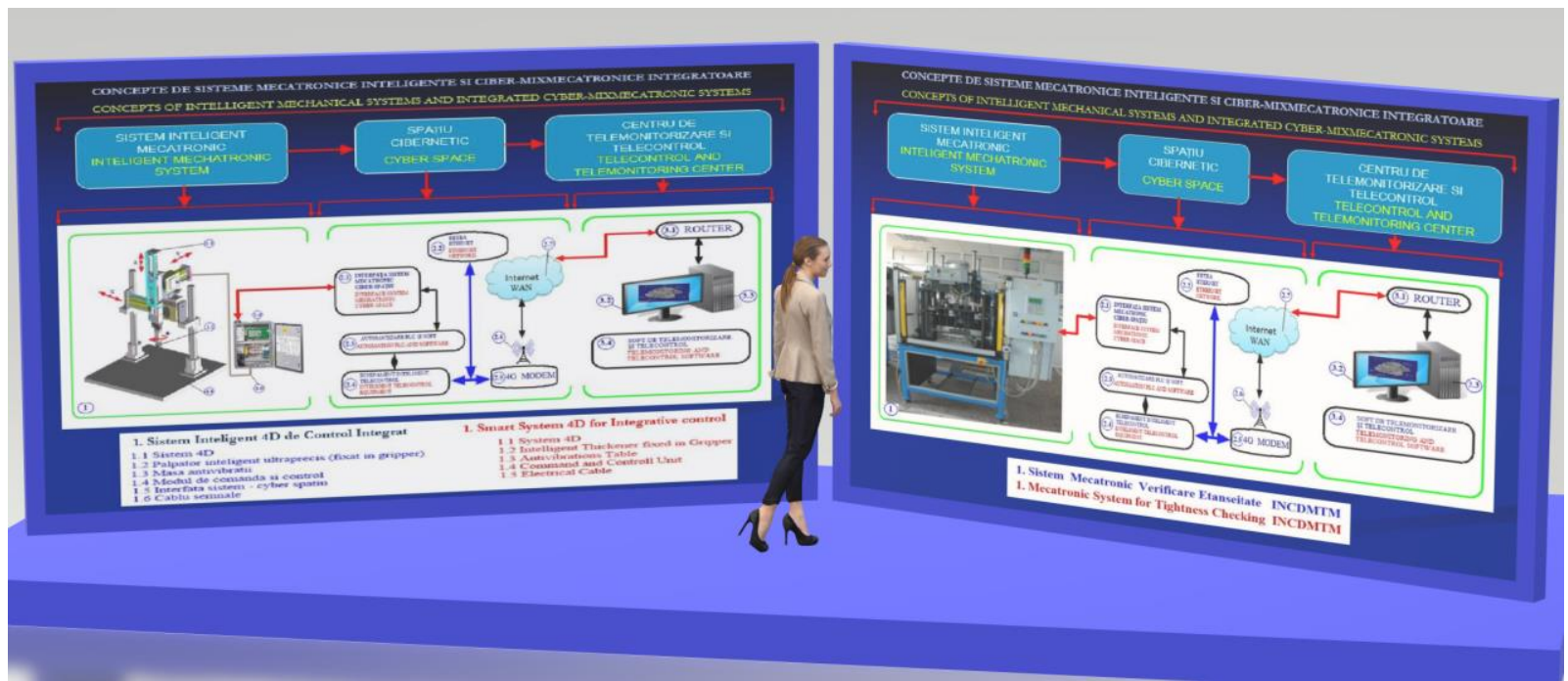

Fig. 1. COBOT-type Platform for Integrated Verification and Control Processes in the Digitized Enterprise and Industry 4.0 in the MixMecatronic field.

cyberpaths and the architecture of telemonitoring teleservice - teleconfiguration and telecontrol centers and with a very important role in collaborative activities with intelligent 4D mechatronic systems and leakproofness verification, the collaborative man, highlighting in fact the technological "cobot".

- in figure 2: The COBOT-type platform for manufacturing (welding and handling parts) from the digitized enterprise and Industry 4.0 in the field of machine building.

The COBOT-type platform for manufacturing processes (welding and handling parts) is logically structured on the welding robot architecture and the parts handling robot (welding robot / robot handling parts/welding robot interface system/robot interface handling parts/mechatronic system for control and control robot welding/mechatronic system for control and control robot handling parts / etc./),

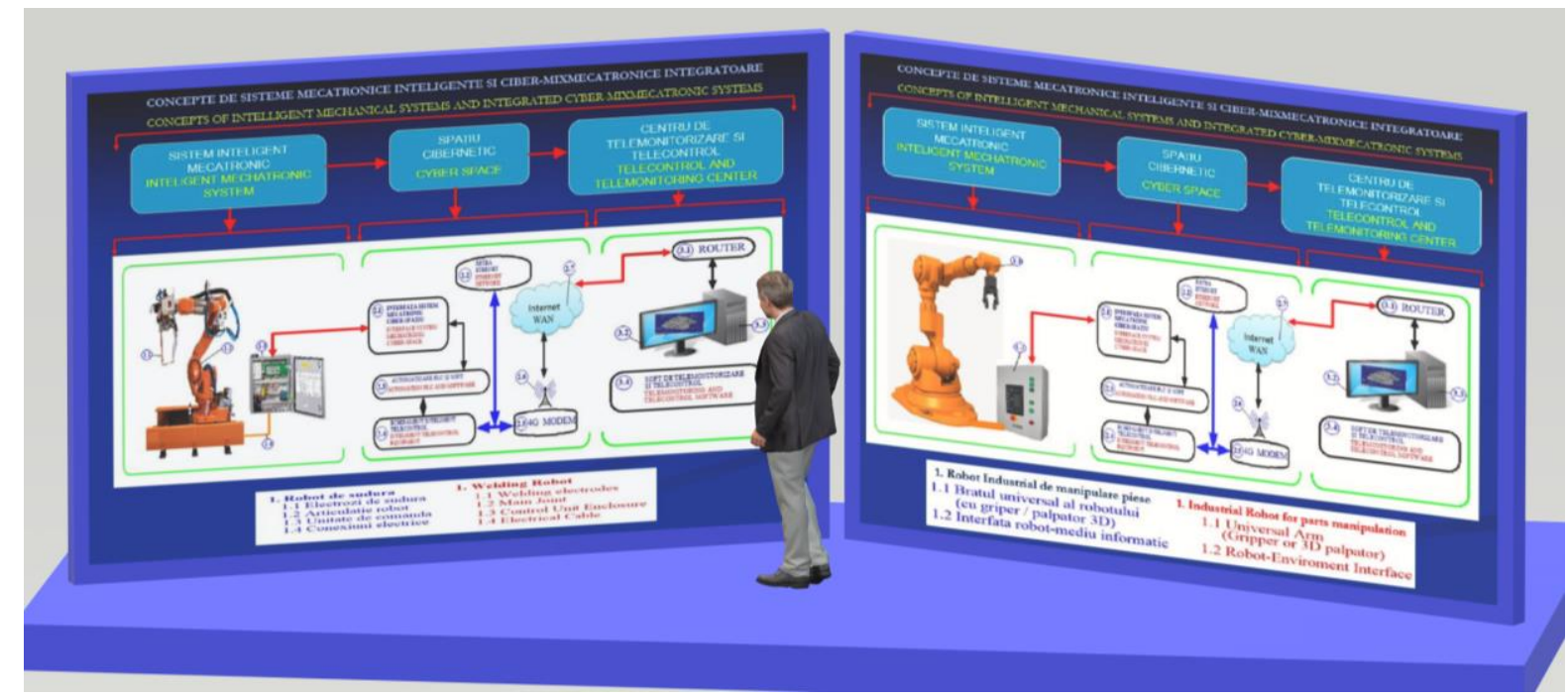

Fig. 2. COBOT-type platform for manufacturing (welding and handling parts) of Enterprise and Industry 4.0 in machine building

- in figure 3: The COBOT-type platform for CNC Machine-Machine Tooling processes in Enterprise and Industry 4.0 in Machine Tools-Building.

The COBOT Platform for CNC Machine Assistance is logically built / structured on the architecture of intelligent mechatronic systems - Assistant Robot
Machine Tools (Industrial robots specific to CNC MU assistance / interfaces between industrial robots and cyber-working spaces for industrial robots) on the architecture of the workspaces for these industrial robots and on the architecture of the monitoring, telemonitoring, servicing, telecontrol and telecontrol centers (routers / computers). 
Cyber-Mixmechatronics Mix Concepts, Integrated in Cobotic Platforms for the Smart Industry (4.0) and the Digitized Enterprise

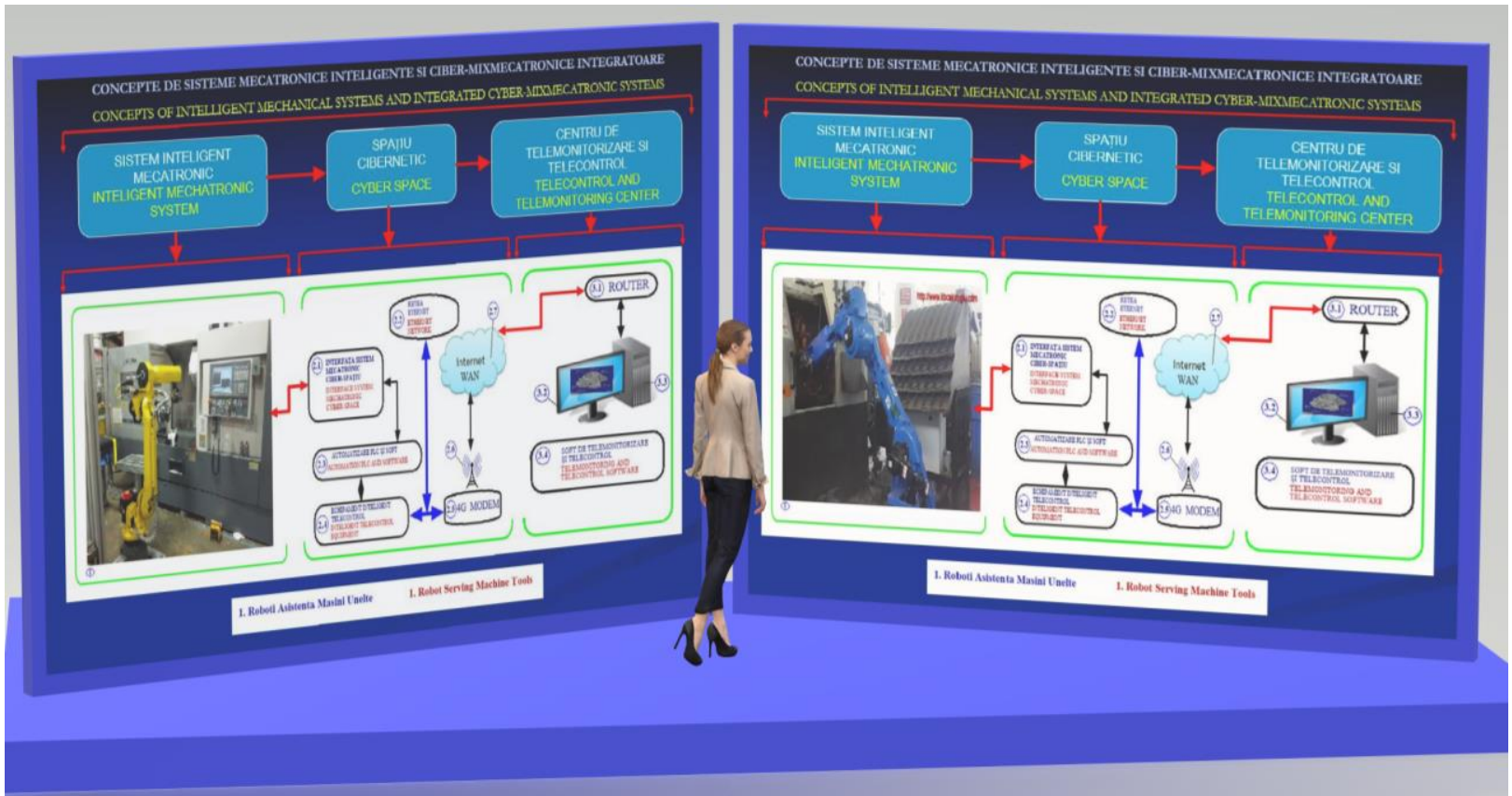

Fig. 3. COBOT-type platform for Assistive Processes Machine Tools with CNC Enterprise and Industry 4.0, M.U.

- in figure 4: The COBOT-type platform for intelligent measurement and control processes in the digitized enterprise and Industry 4.0 in the field of Technology Equipments.

The COBOT-type platform for intelligent dimensional measurement and control is logically structured on the dimensional control mechatronic robot architecture (intelligent mechatronic systems like control robots / control robot interfaces cybernetic spaces / intelligent mechatronic systems for managing and coordinating processes / support systems for control robots / digital touch probe systems for measurement and control / mechatronic systems for operating and collaborating with the robot operator (the collaborative man), on the architecture of cyberspace working and collaboration with the digitized enterprise and on the architecture of monitoring and telemonitoring centers, teleconfiguration, service - teleservice and control and telecontrol (routers / computers / etc.) and collaboration with collaborative human with control robots (special programs for measuring and controlling processes / on special software for measurement and control / human collaborative, etc.).

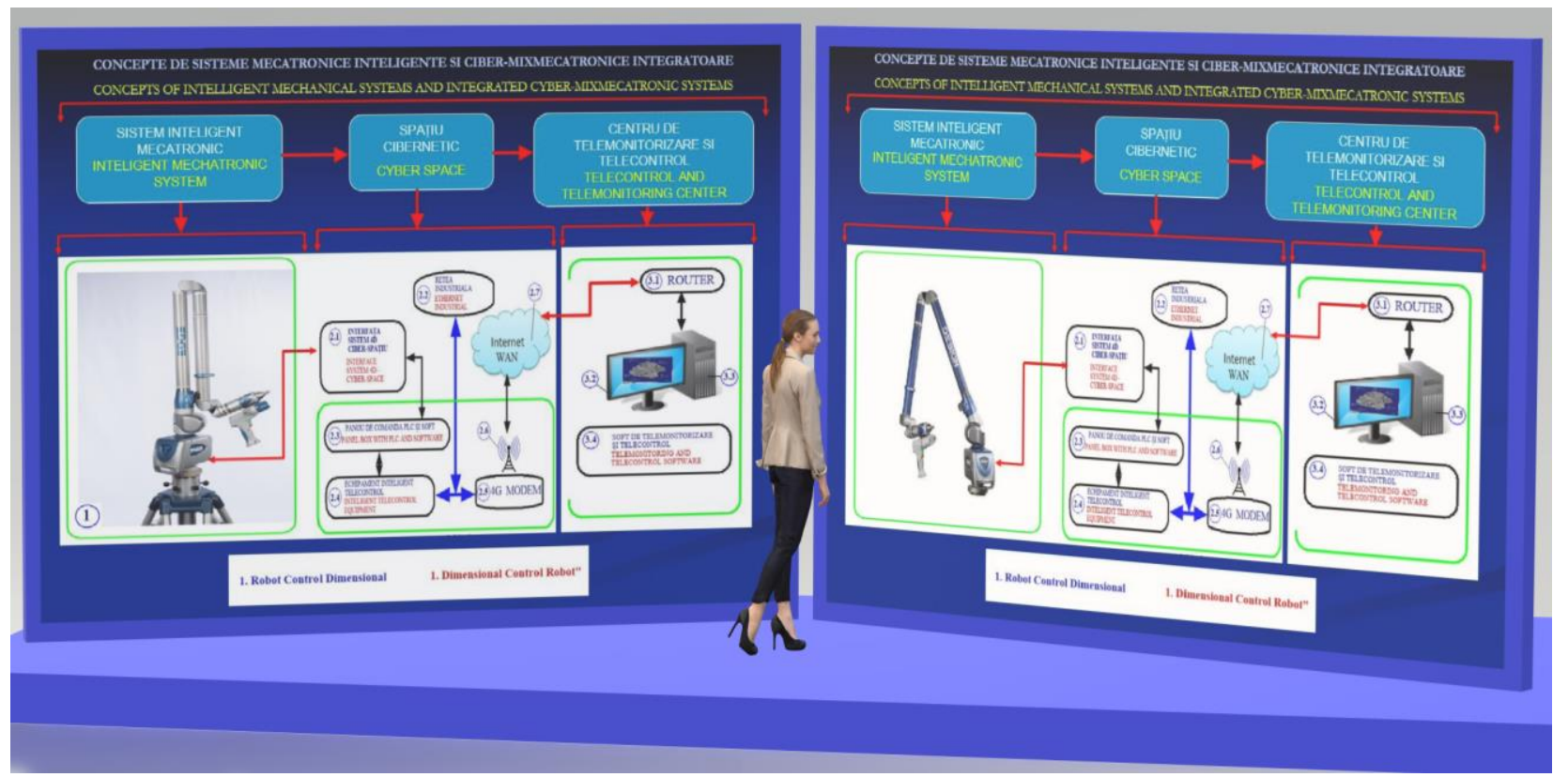

Fig. 4. COBOT-type platform for Smart Measurement and Control Processes in Enterprise and Industry 4.0, Technology Equipment 
- in figure 5: The COBOT-type platform for surgical operations and laboratory analysis (for diagnosis of medical treatments and services) in the

\section{Intelligent Medical field}

The COBOT-type platform for surgical operations and laboratory analyzes is structured on the architecture of the medical surgical robot (DaVinci), a smart medical robot / medical related instrument / mecatronic interface / mecatronic system of operation and coordination / mecatronic intelligent intelligence system surgical operations / mechatronic system for precise and precise manipulation of medical instrumentation / mechatronic intelligent warning and alarm system / mecatronic TV transmission system from "clean space" intended for "surgical operations" / etc.), on the architecture of the robot handling and servicing (robot proper / intelligent haptic systems / control unit / electrical connection systems / space drives / mechatronic auxiliary and adaptive systems to medical devices, etc.), the architecture of the medical robots working spaces (medical interface - cyber space / cyber space for each medical robot / antenna / 4G modem / Internet WAN / Intranet / etc. ), on the architecture of the monitoring centers telemonitoring / service - teleservice / control telecontrol / configuration - teleconfiguration (routers, computers, programs for surgical and medical analyzes, special software, etc.) (the collaborative human of the platform).

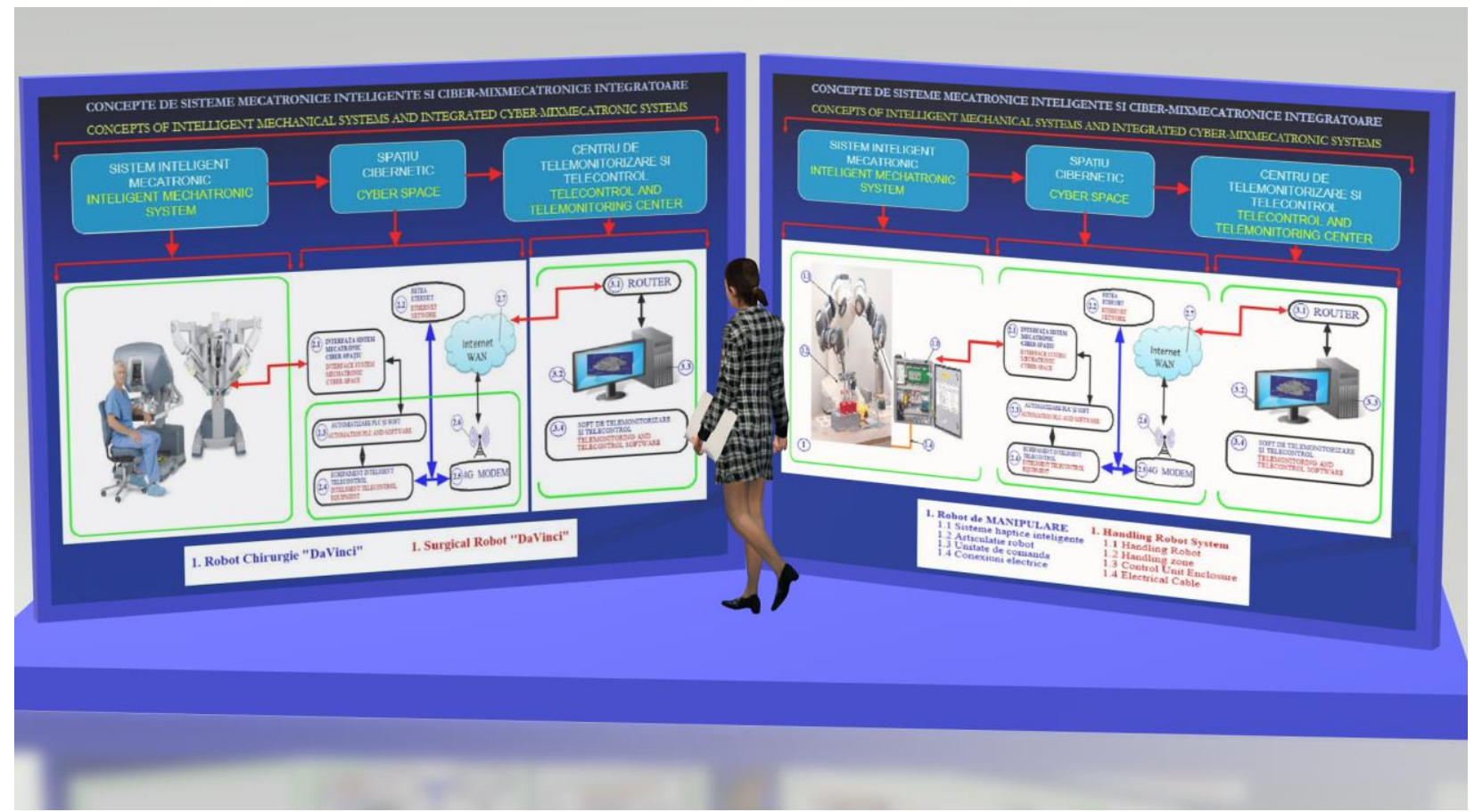

Fig. 5. COBOT-type platform for surgical operations and laboratory analyzes in the field of Intelligent Medical

- in figure 6: The COBOT-type platform for Patient Analysis and Walking Recovery in Intelligent Medical and Biomedical field

The COBOT-type platform for Patient Walking through Analysis and Recovery is structured and built on the Intelligent Walking through Analysis Mechatronic System architecture (the actual stripe / warning and alarm system / stop-lock system / individual operating system / etc.) on the mechatronic track gauge architecture (the actual band / the computerized system for medical recovery parameters / warning and alarm system / stop-lock / individual operating system / etc.) on (4GMODEM / Antenna / Internet WAN / Intranet, etc.), on the architecture of telemonitoring / teleservice / telecontrol / teleconfiguration centers (routers, computers, software and software), the architecture of the cybernetic spaces of the two intelligent mechatronic systems of Analysis and Walkback Recovery Specialized Analysis and Recovery / etc.). 


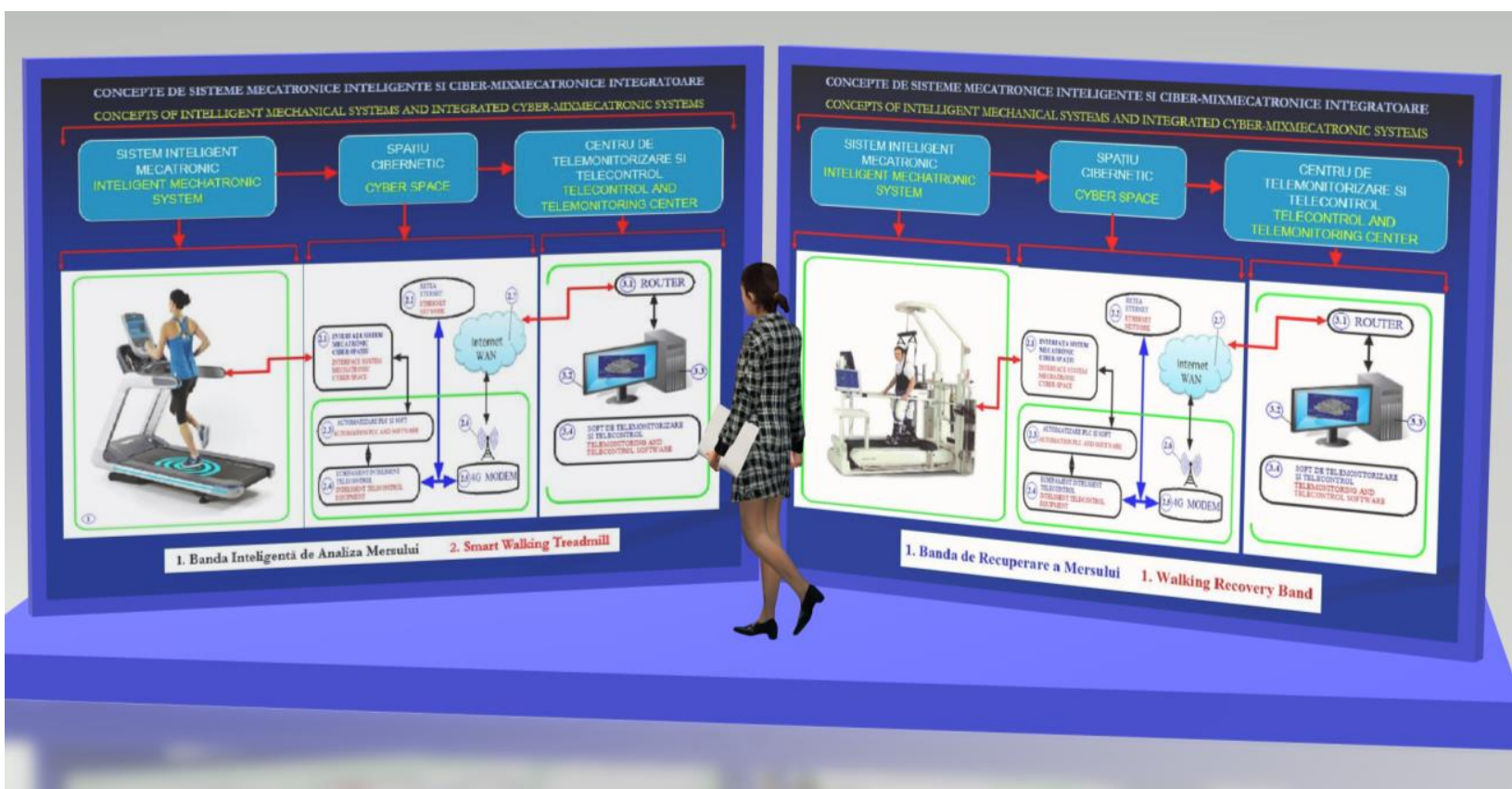

Fig. 6. COBOT-type platform for Walking through Analysis and Recovery in Intelligent Medical and Biomedical

- in figure 7: The COBOT-type platform for control and automotive manufacturing processes in the Enterprise and Industry 4.0 Automotive industry.

The COBOT-type platform for Intelligent Manufacturing in Intelligent Control Processes is built and structured on the Automobile Robot Architecture for Control (Intelligent Operating Mechronic Intelligent Operating System / Intelligent Alarm and Alarm System / Mechatronic Coordinating System and control / cyber-interfacing system / intelligent self-adjusting and selfpositioning system / etc.), on the robot architecture of automotive casting control (engine / gearbox / injection pump / etc.) Intelligent control / Mechatronic Intelligent Operating System / Intelligent Alarm and Alarm System / Mechatronic Coordination and Control System / Mechatronic Coordinating and Command System with Measuring Metering / etc.), Cyberspace Architecture (Interface between control and external cyberspace / Internet WAN / Intra net / 4GMODEM / etc.), on the collaborative program architecture of the IT operator (collaborative man with control robots) and on the architecture of the telemonitoring centers - teleconfiguration - teleservice - telecontrol (routers, computers / measurement / control / analysis / operational decisions).

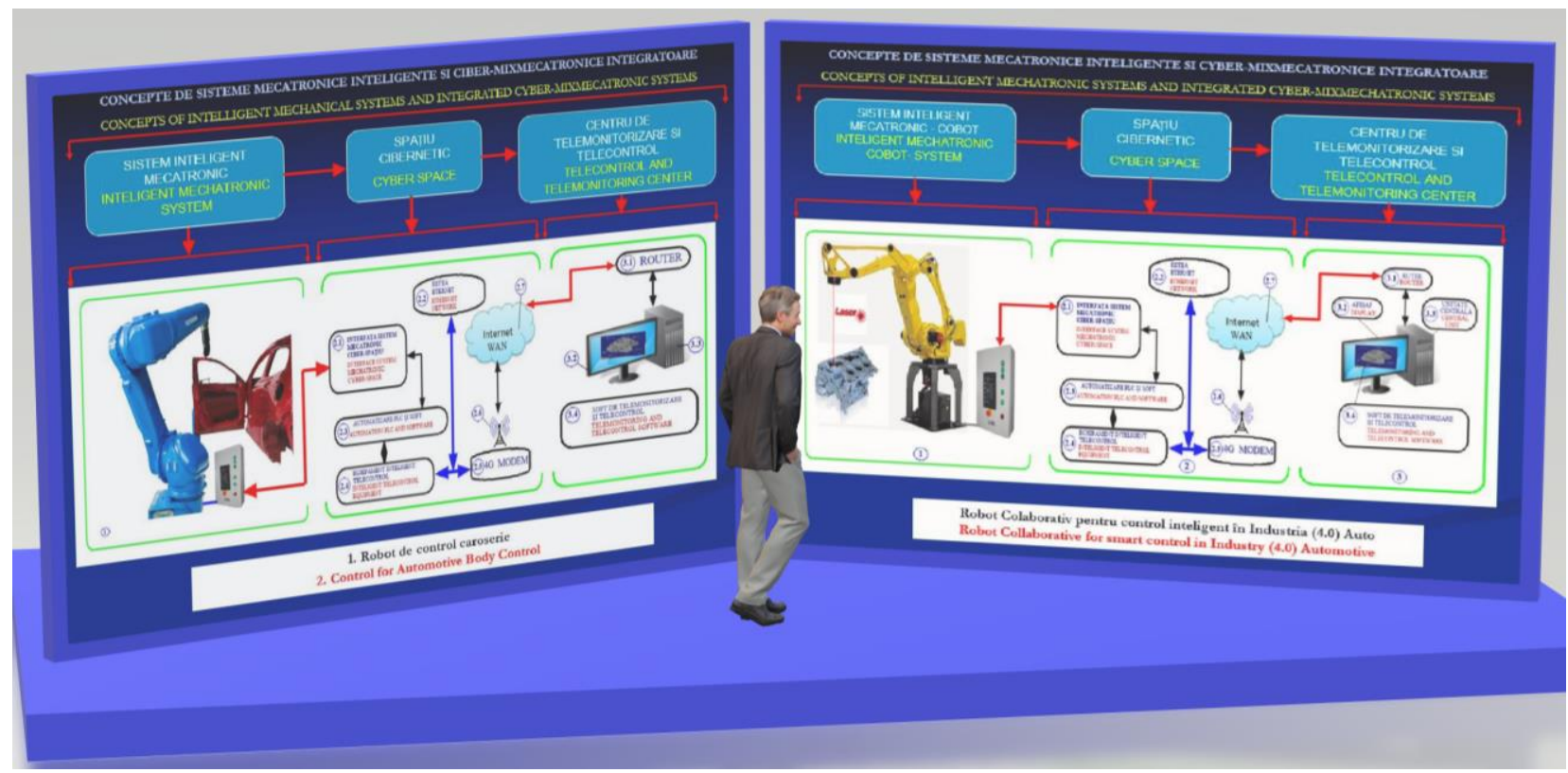

Fig. 7. COBOT-type platform for control processes and manufacturing lines in Enterprise and Industry 4.0 
- in figure 8: a COBOT-type platform for social services in the Intelligent City

The COBOT social services platform is built and structured on Drone's architecture for filming (Electronic Drone / Fastener Film / Camera / Travel and Transport Controller / Special Software for Monitoring / Configuration / Control / Systems antishock / protection systems for wind turbines / etc.), on the parcels' drone architecture (electronic drones / parcel shelf / camera / controllers for transport and transport / special software for monitoring / anti-shock systems / wind turbine protection systems, etc.) and the collaborative program architecture of the drone operator / operator (collaborative man with Electronic Drone).

Electronic drones are equipped with interfaces between drones and cyberspace (4GMODEM / antennas / interfaces / etc) as well as between remote control and remote control centers, telecontrol and teleconfiguration (interfaces / routers / computers / programs - specialized software/.

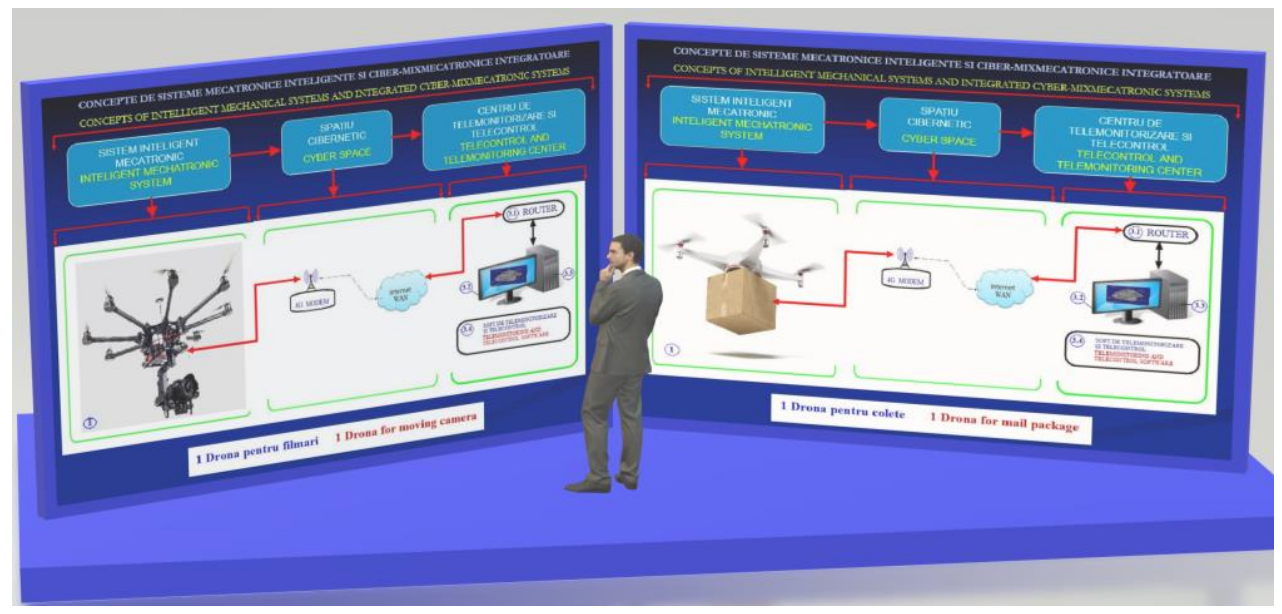

Fig. 8. COBOT-type platform for Social Services in the Intelligent City

- in figure 9: The COBOT-type platform for agricultural processes in the field of Intelligent Agriculture

The COBOT-type platform for agricultural processes is structured and built on the architecture of the mobile sprinkler mobile robot (the actual robot / agricultural instrument set / monitoring controller / specialized software / drive systems for agricultural services / intelligent mechatronic control systems and coordination), on the architecture of the intelligent system for the agricultural environment (smart system itself / instrument set for agricultural services / intelligent coordination and control system / interface for cyberspace / etc.), on the architecture of the cybernetics the mobile mobile robot and the intelligent mecatronic system for agricultural services ? (antennas / 4GMODEM) / Internet WAN / Intranet / etc.), the architecture of the monitoring / telemonitoring, configuration / teleconfiguration and control / telecontrol centers (routers, programs - specialized software / etc) and hand architecture operator geriatric for collaboration with the two agricultural systems (the collaborative man of the COBOT platform).

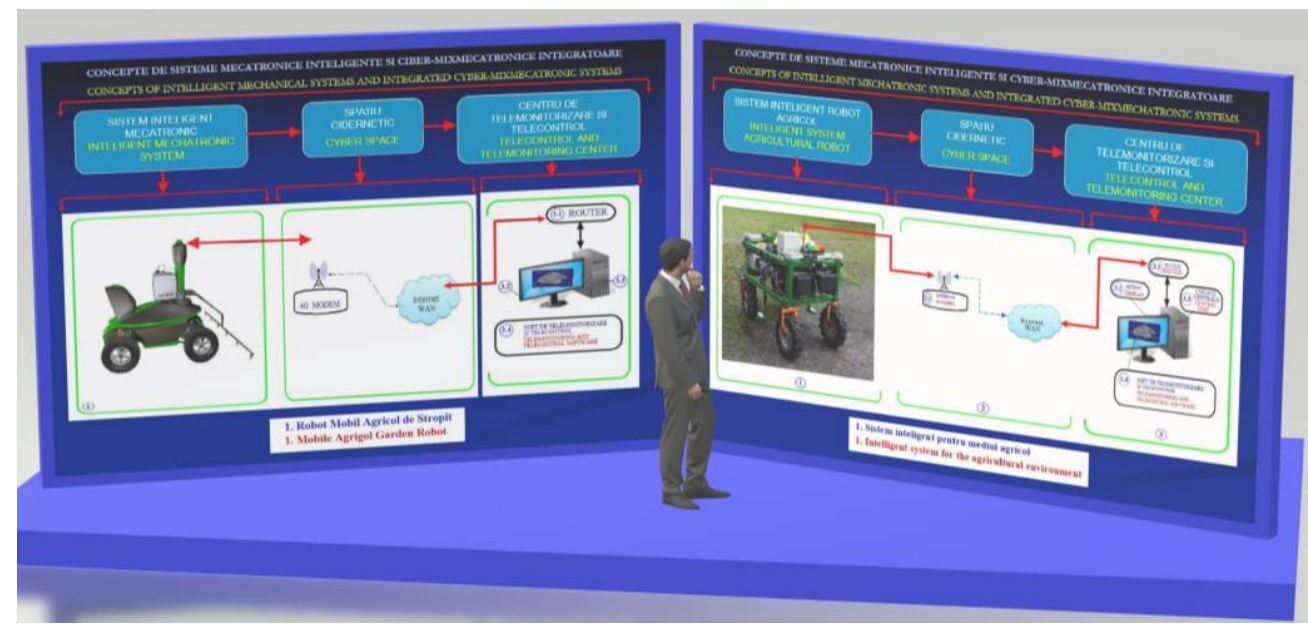

Fig. 9. COBOT-type platform for agricultural processes in Intelligent Agriculture 
- in figure 10: The COBOT-type platform for industrial assembly processes from the Digitized Enterprise and Industry 4.0 in the field of Machine Building

The COBOT-type platform for industrial control processes is structured and built on the collaborative robot architecture for intelligent industrial control of the geometry of the space where another industrial benchmark is to be assembled (the actual robot / packet of prehensive devices, mechatronic operating system and coordination / interfacing with cyberspace / etc.), on the intelligent robot architecture for automotive windscreen assembly (the actual robot / instrumentation and control set / mechatronic system for operation and coordination / interfacing with cyberspace / programs specialized software / etc), on the operator's management architecture (through programs and softwares), the collaborative man (with the two control and installation robots), on the architecture of the antenna spaces / 4GMODEM) / Internet WAN / Intranet / etc.) and on the architecture of the monitoring centers has / telemonitoring, configuration / teleconfiguration, control / telecontrol, etc. (routers, computers / programs specialized software / etc).

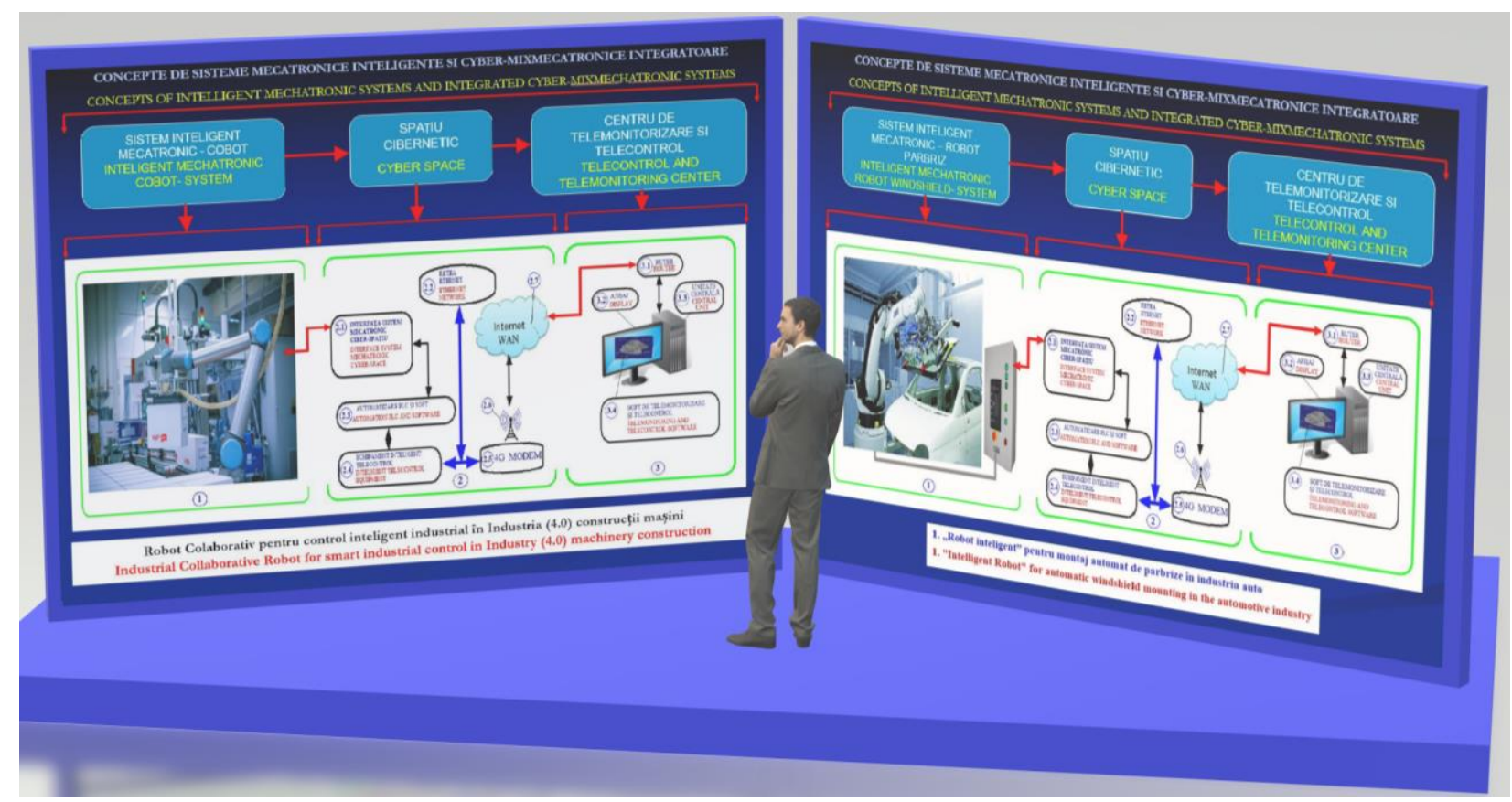

Fig. 10. COBOT-type platform for assembly and industrial control in Enterprise and Industry 4.0, Machine Building

- in figure 11: The COBOT-type Platform for Intelligent Control Processes in the Digitized Enterprise and Industry 4.0 in the Electronics and Electrotechnics field

The COBOT-type Platform for Intelligent Control Processes in the Electronics and Electrotechnics field is structured and built on the collaborative robot architecture - the COBOT for intelligent laser control (the robot itself / mechatronic precision positioning system of the electronic marker ) / Intelligent operation and coordination mechatronic system / Mechatronic interfacing system with cyberspace / laser related system / control system viewing system / related system with measuring and control instrumentation etc.), on the collaborative robot architecture - COBOT Intelligent Laser Control (the robot itself / the mechatronic precision positioning system of the electronic circuit board (PCB) / intelligent operation and coordination mechatronic system / cybernetic interfacing system / laser related system / visualization of the measurement process (4GMODEM / Antennas / Internet / Intranet / etc.) and on the architecture of the telemonitoring, teleservice, teleconfiguration and telecontrol centers (the collaboration of the robots), the architecture of the cyberspace routers / computers / programs specialized software / etc). 


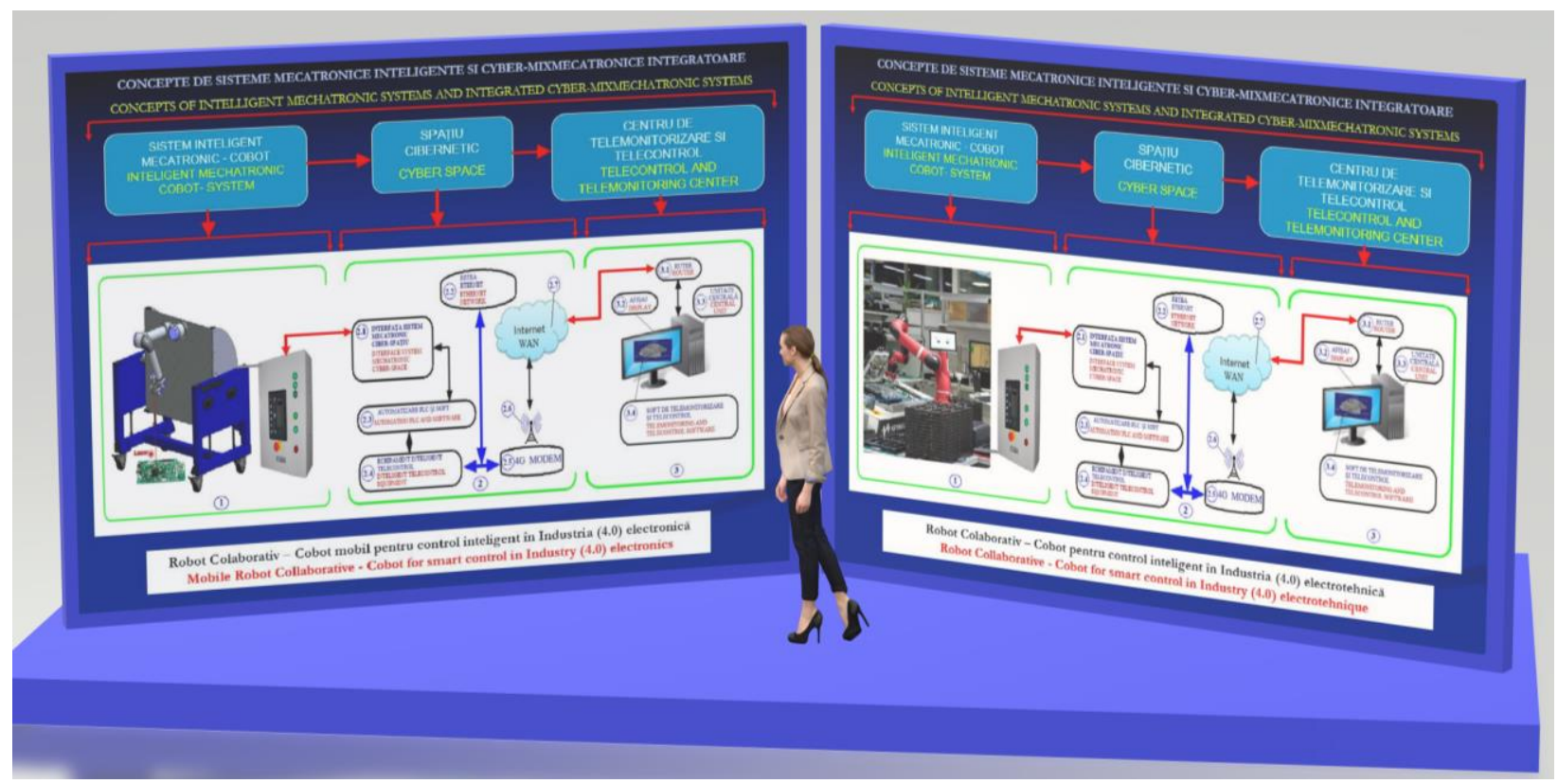

Fig. 11. COBOT-type Platform for Intelligent Control Processes in Enterprise and Industry 4.0, in Electronics and Electrotechnics

- in figure 12: The COBOT-type platform for intelligent manipulation and control processes in the Digitized Enterprise and Industry 4.0, in the Mechatronics and Cyber-MixMechatronics

The COBOT-type platform for intelligent handling and control is built and structured on the collaborative robot architecture - COBOT for intelligent manipulation (the actual robot / Mechatronic control and operation system / mechatronic viewing system / cybernetic interfacing system / the system for handling devices / etc.), on the collaborative robot architecture - COBOT for intelligent control (the robot itself / smart mechatronic interface for cyberspace / command and operation system / intelligent viewing system / system (4GMODEM / antennas / Internet / Intranet / etc.), on the architecture of the monitoring centers, configuration, remote control (routers / computers / software - etc.) and on the collaborative management architecture of the operation (the collaborative man of intelligent robots), without which the COBOT platform can not function, the human-robot collaboration being mandatory and deployed according to an appropriate structure of the COBOT platform software.

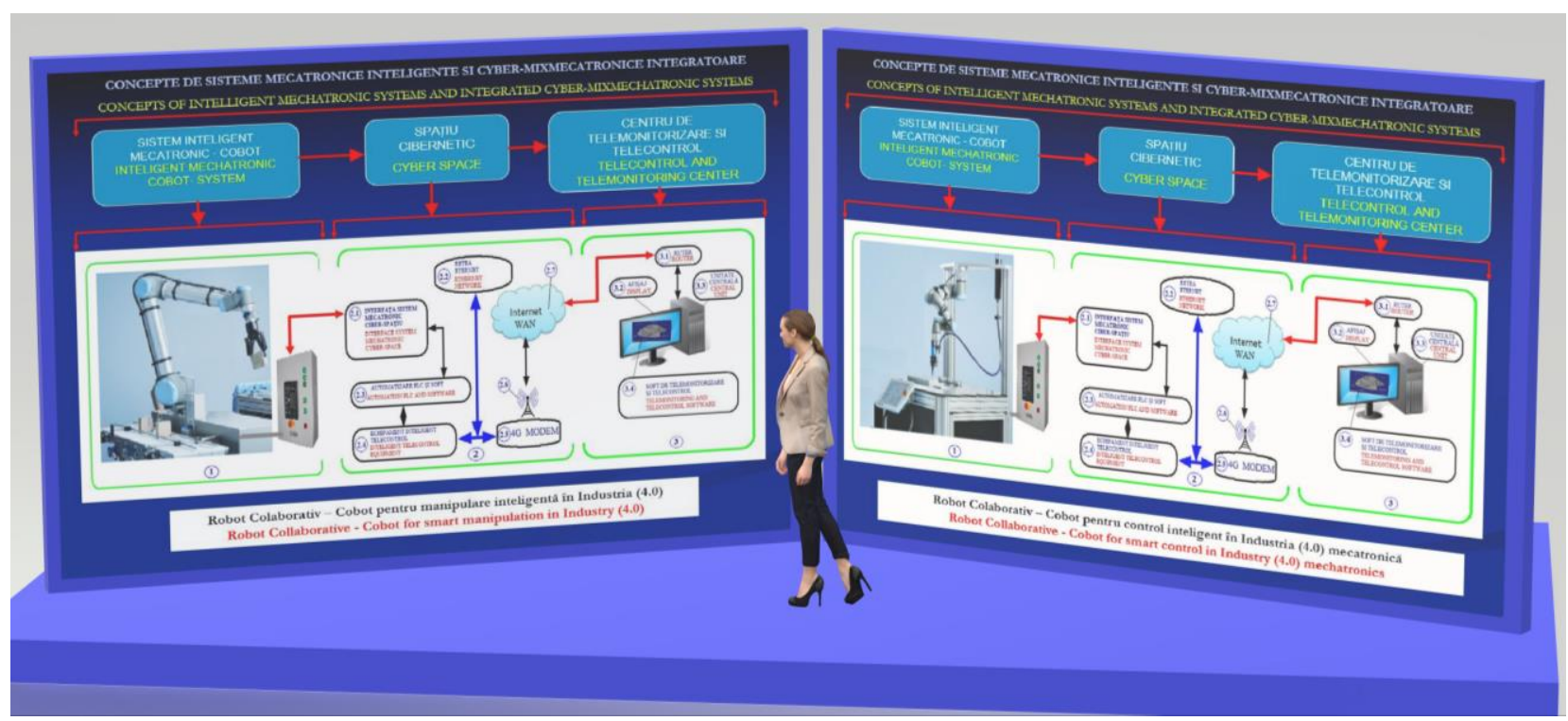

Fig. 12. COBOT-type platform for Intelligent Handling and Control Processes in Enterprise and Industry 4.0 in the Mechatronics and Cyber-MixMechatronics field 
- in figure 13: The COBOT-type platform for industrial control processes from the Digitized Enterprise and Industry 4.0 in the Aeronautics field

The COBOT-type platform for aeronautical industrial landmark control processes is built and structured on the collaborative control robots architecture of aeronautical industrial landmarks (robots / mechatronic operating and control systems / mechatronic viewing systems / mechatronic interfaces with cyber spaces / etc.), on the architecture of cyberspace (4GMODEM / Antennas / Internet / Intranet / IoT / CPS / CMS / etc.), on the architecture of monitoring / telemonitoring, service / teleservice, control / telecontrol and teleconfiguration (routers / computers / programs specialized software / etc.) and collaborative management architecture (the collaborative man with the technological platform, where the humanrobot collaboration process defines COBOT and the obligation through the technological platform.

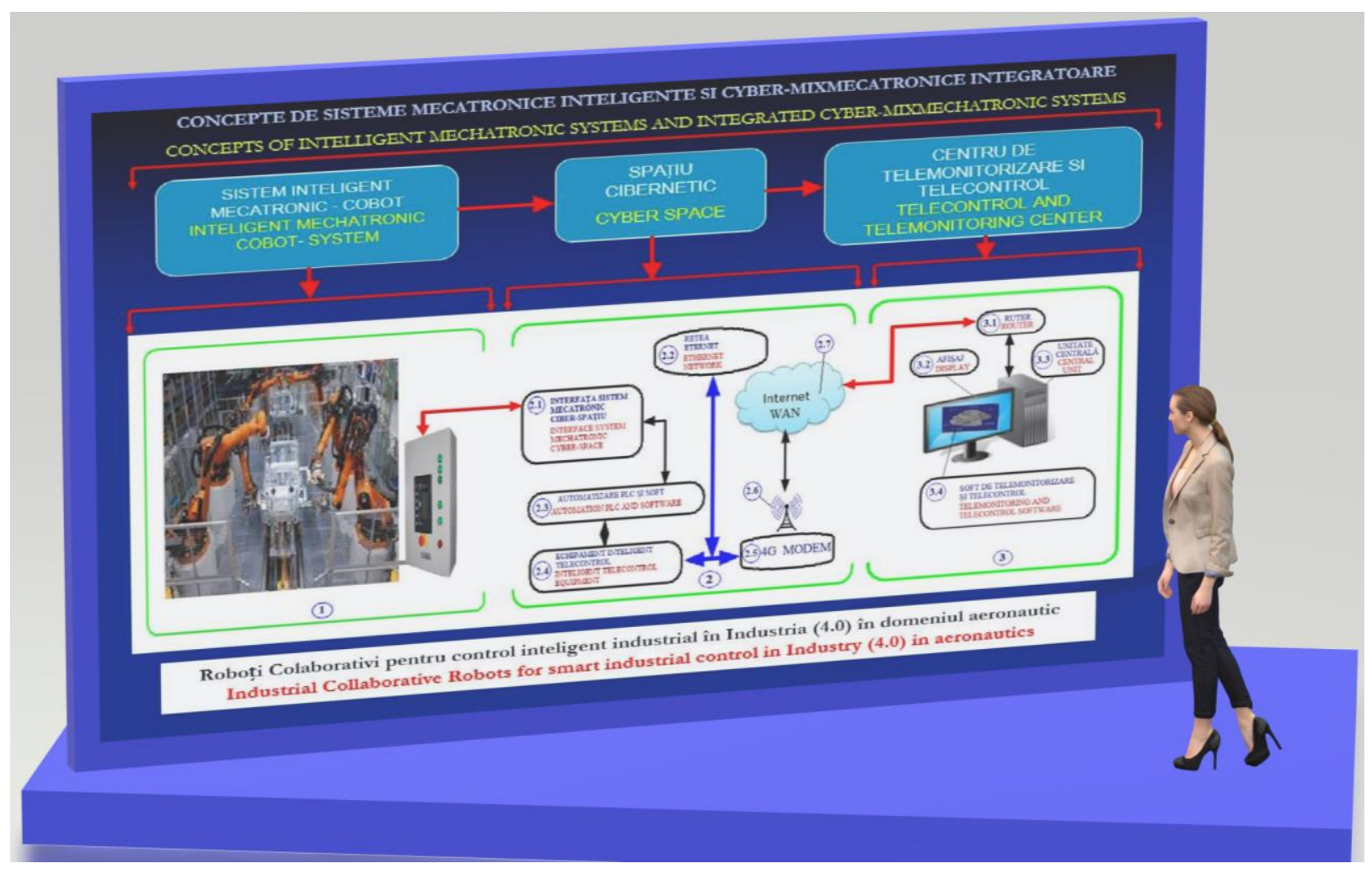

Fig. 13. COBOT-type platform for industrial control processes in Enterprise and Industry 4.0 Aeronautics

- in figure 14: The COBOT-type platform for positioning processes in Metrology Laboratories in Enterprise and Industry 4.0, in the field of Intelligent Metrology

The COBOT-type platform for positioning processes in metrology laboratories is built and structured on the collaborative robot architecture COBOT for intelligent positioning (the intelligent robot with 12 axes - 6 Cartesian and 6 polar proper / drive system for each 12 axes / robot movement visualization system / intelligent operating and coordinating system / intelligent integration system sensors and transducers for precise and accurate measurement / positioning processes / etc), on the collaborative robot architecture - COBOT for intelligent positioning (Intelligent 6-Axis-3 Cartesian and 3-Axle Robot / Drive System for Each of the 6 Axes / Robot Movement Viewing System / Intelligent Operating and Coordinating System / Intelligent Integration System Sensors and Transducers for Measurement / Positioning Processes precise and very precise / etc.), per country (antennas / 4GMODEMs / Internet / Intranet / interfacing systems / etc), on the architecture of monitoring / telemonitoring, configuration / teleconfiguration, control / telecontrol and service / teleservice centers (routers / computers / programs specialized software / etc). 


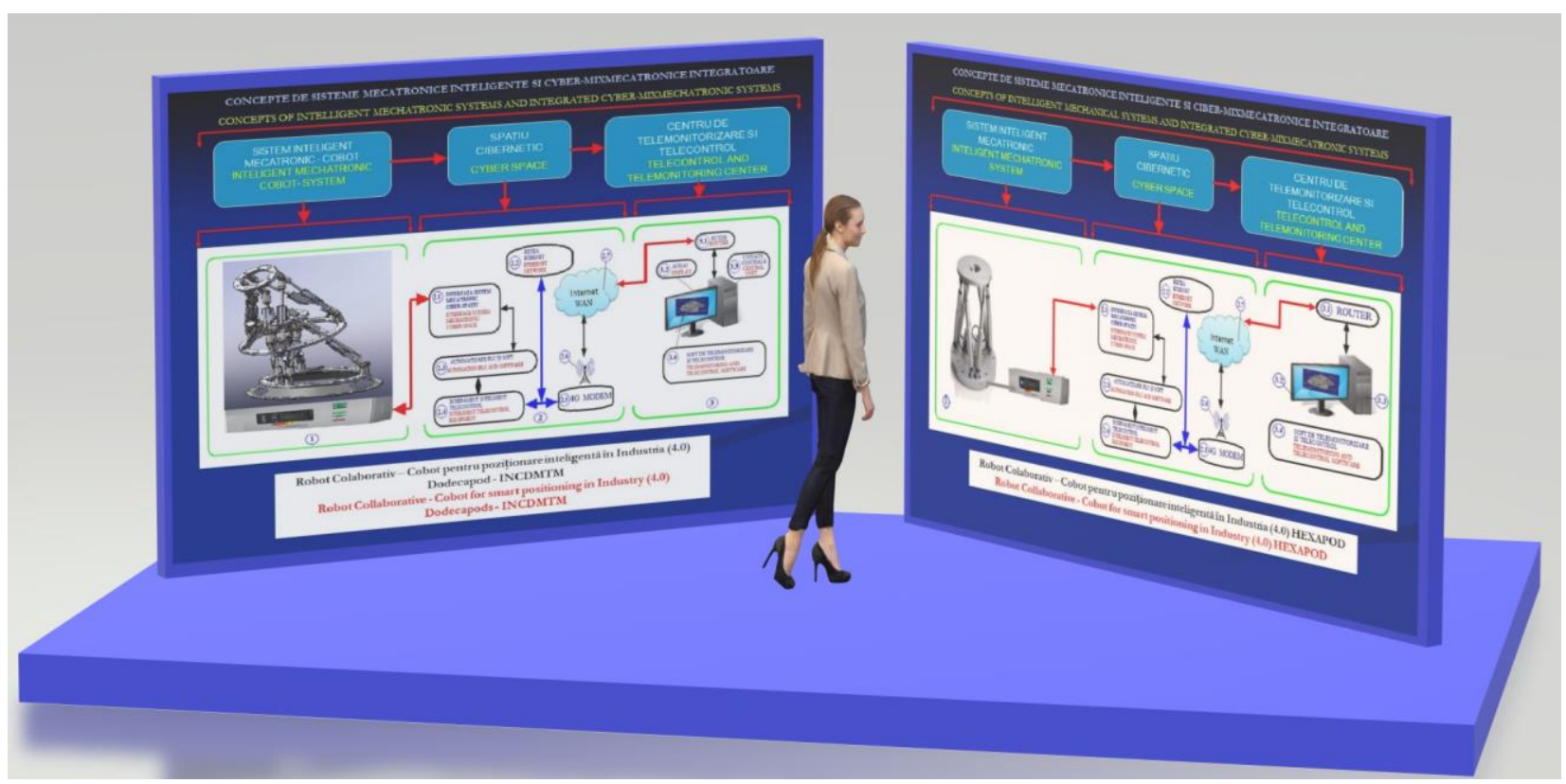

Fig. 14. COBOT-type platform for positioning processes in metrology laboratories in Enterprise and Industry 4.0

\section{Conclusions}

The scientific paper shows that industrial digitization will impact both horizontally and vertically on the value chain, which implies that on the one hand companies need to integrate and digitize their data flow vertically. to the development of products and purchases to processing and transport logistics, and on the other hand, involves a horizontal collaboration with key suppliers, customers and other partners in the value chain

Companies, businesses and industry in general Industry 4.0 should be involved in the implementation and deployment of complex digital solutions, and all committed staff are fully confident that industrial digitization is the most appropriate and necessary choice for the future.

The scientific work also synthesizes the beginnings of industrial digitization by introducing intelligent concepts and solutions proposed by the author for mechatronic and cyber-mixmechronic integrated systems that are or are to be implemented in different industrial sectors (automobiles, aerospace, agriculture, medicine, etc.) from Romania.

The Enterprise and Industry Digitization Strategy 4.0 is synthesized at national and European level in the following chart:

\section{Pillar 1}

Single Digital Market - Free cross-border access to online services and information

\section{Pilllar 2}

Interoperability and standards - integration, devices, applications, data and services in the code of social ethics

Pilllar 3

Trust and security - Increase Internet users' trust in electronic services and online transactions through transparency and security

Pillar 4

Fast and ultrafast access to the Internet - aims to invest in infrastructure in broadband equipment

\section{Pillar 5}

Research and Innovation in ICT - Stimulates adequate funding for increased competitiveness

\section{Pillar 6}

Increasing the digital literacy of skills and inclusion - Creating a bridge to the digital divide

Pillar 7

ICT benefits for EU society - ICT's ability to reduce bureaucracy, support elderly care, improve health services, and deliver public services

Goals to be achieved by 2020 are:

- Employment (75\% of people between 20 and 65 years should be employed)

- Research / Development (3\% of GDP should be allocated to R / D)

- In the field of education (40\% of people between 30 and 34 years to complete the third level of education)

- Combating poverty and social exclusion.

\section{References}

[1] German Engineering Association (www.vdma.org), German association of ICT industry (www.bitkom.org), and German association of electrics and electronics industry (www.zvei.org);

[2] Club IT\&C - TAG Media - mai 2018: Digital Transformation 2018, ISSN 1583-5111.

[3] FU și Acatech (2013) Asigurarea viitorului industriei prelucrătoare din Germania - 
Recomandări pentru implementarea iniţiativei strategice INDUSTRIE 4.0 - Raport Final;

[4] Gheorghe, I. G., Constantin, A, Ilie, I. Mechatronics and Cyber Mechatronics in Intelligent Applications from Industry and Society , Proceedings of OPTIROB 2016, Romania;

[5] Gheorghe, I. G., Mecatronica și CyberMixMecatronica în Industria 4.0, Editura CEFIN, ISBN: 978-606-8261-28-7, Ed. CEFIN, 2018;

[6] https://pt.dreamstime.com/ilustra\%C3\%A7\%C3 \%A3o-stock-agricultura-esperta-image83848448

[7] http://digital-agri.fr/agriculture-etdigitalisation/

[8] http://electronica-azi.ro/2017/07/07/industrie4-0-realitatea-interconectata-a-fluxurilorindustriale-de-productie/

[9] https://media.hotnews.ro/media server1/docu ment-2018-10-11-22752549-0-studiuguvernare.pdf

[10] Boston Consulting Group (2015) Industria 4.0: Viitorul productivității și creșterii în industria prelucrătoare

[11] BMWi (2016) Digitizarea industriei - Plattforma Industrie 4.0, Raport de activitate - Aprilie 2016

[12] Varela, M.L.R., Putnik, G.D., Manupati, V.K., Rajyalakshmi, G., Trojanowska, J., Machado, J. (2018) Collaborative manufacturing based on cloud, and on other I4.0 oriented principles and technologies: A systematic literature review and reflections

[13] Management and Production Engineering Review, 9 (3), pp. 90-99, DOI: $10.24425 / 119538$

[14] Barros, C., Leão, C.P., Soares, F., Minas, G., Machado, J. (2013) RePhyS: A multidisciplinary experience in remote physiological systems laboratory International Journal of Online Engineering, 9, pp. 21-24, DOI: 10.3991/ijoe.v9iS5.2756

[15] Karnouskos, S.; Colombo, A.W.; Bangemann, T.; Manninen, K.; Camp, R.; Tilly, M.; Stluka, P.; Jammes, F.; Delsing, J.; Eliasson, J. “A SOA-based architecture for empowering future collaborative cloudbased industrial automation", IECON 2012 - 38th Annual Conference on IEEE Industrial Electronics Society, 2012 , Page(s): 5766 - 5772.

[16] Gheorghe Gheorghe, "Original concepts and achievements for designing of smart mechatronic and cyber-mixmechatronic systems used in laboratories and in the industry", 18th IFAC Conference will take place by support of IFAC (International Federation of Automatic Control), TECIS 2018, 13-15 september, Baku, Azerbaijan, publicata de IFACPapersOnLine. 